Int. J. Dev. Biol. 58: 767-774 (2014)

doi: $10.1387 / \mathrm{ijdb} .140101 \mathrm{jk}$

\title{
Control of timing of embryonic M-phase entry and exit is differentially sensitive to CDK1 and PP2A balance
}

\author{
MOHAMMED EL DIKA ${ }^{1,2}$, DAMIAN DUDKA ${ }^{1,2}$, CLAUDE PRIGENT11,2, JEAN-PIERRE TASSAN ${ }^{1,2}$, \\ MALGORZATA KLOC ${ }^{3}$ and JACEK Z. KUBIAK ${ }^{1,2}$ \\ ${ }^{1}$ CNRS, UMR 6290, Institute of Genetics and Development of Rennes, Cell Cycle Group, Rennes, France, \\ 2University Rennes 1, UEB, IFR 140, Faculty of Medicine, Rennes, France and \\ ${ }^{3}$ The Houston Methodist Research Institute, Houston, TX, USA
}

\begin{abstract}
Harmonious embryo development requires precise coordination between the timing of the cell cycle and the developmental program. Cyclin accumulation determines the timing of the cell cycle M-phase entry and its degradation determines the timing of the M-phase exit. It is well known that CDK1 and PP2A also govern M-phase entry. However, it is unknown how this kinase and phosphatase regulate the precise timing of events at the beginning of the M-phase and how they cooperate with cyclin metabolism. Here we use Xenopus laevis one-cell embryo cell-free extract experiments to answer this question critical for understanding the regulation of embryo development. Using, separately, low concentrations of the chemical inhibitor of CDK1, RO3306 (RO), or the inhibitor of phosphatases, okadaic acid (OA), we show that moderately diminished CDK1 or PP2A activities results in a delay and an acceleration respectively, of M-phase entry. Simultaneous diminution of CDK1 and PP2A activities results in an intermediate timing of M-phase entry, prolongs the duration of $\mathrm{M}$-phase and diminishes the dynamics of cyclin B2 degradation. We thus show, for the first time, that equilibrium between CDK1 and PP2A specifies the timing of M-phase entry and exit and regulates the dynamics of cyclin B degradation upon M-phase exit in Xenopus laevis first embryonic mitosis.
\end{abstract}

KEY WORDS: cell cycle, cyclin, PP2A, CDK, M-phase entry

\section{Introduction}

The protein phosphorylation is crucial for controlling entry into and exit from mitosis. The key molecular event that initiates mitosis is activation of Cyclin-Dependent Kinase 1 (CDK1). CDK1 activation requires binding of a regulatory subunit - cyclin. Cyclins accumulate gradually during interphase and during the period of the entry into mitosis. CDK1 is inactivated upon exit from mitosis when it dissociates from the cyclin (Nishiyama et al., 2005). The functional interactions between CDK1 and PP2A are crucial for the mitotic entry and exit (Krasinska et al., 2011, reviewed in Fisher et al., 2012). CDK1 activation triggers activation of Greatwall kinase, which phosphorylates ARPP-19, and this in turn inactivates PP2A (Gharbi-Ayachi et al., 2010; Moshida et al., 2010, reviewed in Haccard \& Jessus, 2011). PP2A inactivation during M-phase has a key importance for the mitotic progression because it allows correct accumulation of CDK1 substrates in their phosphorylated forms (Mochida et al., 2009). Upon M-phase exit, CDK1 inactiva- tion allows Greatwall inactivation, ARPP-19 dephosphorylation, PP2A activation and thus dephoshorylation of mitotic substrates necessary to effectively exit from the M-phase. Other studies show that CDK1 inhibits type 1 protein phosphastases (PP1) during mitosis (Wu et al., 2009). At the exit from mitosis, CDK1 is inactivated and this allows PP1 phosphorylation at Thr 320 and inhibitor-1 dephosphorylation at Thr 35 (Wu et al., 2009). Thus, CDK1 remains in a complex interrelationship not only with PP2A but also with PP1, which are the two major okadaic acid-sensitive phosphatases involved in mitotic regulation. Before entry into mitosis, CDK1 activity is inhibited by phosphorylation on Thr 14 and Tyr 15 by Myt1 and Wee1 kinases (Gould \& Nurse, 1989; Parker et al., 1992; Lundgren et al., 1991). CDC25 is the phosphatase assuring Thr14 and Tyr15 dephosphorylation, and therefore, full

Abbreviations used in this paper: CDK-1 Cyclin-dependent kinase 1; OA, okadaic acid; RO, the RO3306 chemical inhibitor of CDK1.

\footnotetext{
*Address correspondence to: Jacek Z. Kubiak. CNRS, UMR 6290, Institute of Genetics and Development of Rennes, Cell Cycle Group, F-35043 Rennes, France. e-mail: jacek.kubiak@univ-rennes1.fr
}

Accepted: 2 December 2014.

ISSN: Online 1696-3547, Print 0214-6282 
CDK1 activation (Kumagai \& Dunphy, 1991; Gautier et al., 1991; Sebastian et al., 1993). During interphase, CDK1/cyclin A accumulates and promotes partial CDC25 activation through PIx1 activation. This initiates CDK1/cyclin B activation, which phosphorylates Serine 285 of CDC25. This, in turn, recruits PP1 and results in higher activation of CDC25. CDK1/cyclin B complex inhibition is then directly removed by dephosphorylation of its two inhibitory residues by CDC25 (Kumagai \& Dunphy, 1991; Gautier et al., 1991; Sebastian et al., 1993). This results in the triggering of an activation loop and immediate and full activation of CDK1/cyclin B complex and the M-phase entry. At the M-phase exit, polyubiquitination of cyclin B by ubiquitin ligase APC/C (Anaphase-Promoting Complex/ Cyclosome) targets CDK1-bound cyclin B to the proteasome 26 $\mathrm{S}$ (Nishiyama et al., 2000). This in turn results in the dissociation of the cyclin B/CDK1 complex, degradation of cyclin B and dephosphorylation, by the type $2 \mathrm{C}$ protein phosphastases (PP2Cs), of Thr161 of CDK1 and its definitive deactivation (Chesnel et al., 2006, 2007). Thus, interplay between CDK1, PP2A and CDC25 is essential for triggering the CDK1 activation loop and M-phase entry. The studies of the role of CDK1 and PP2A in triggering Mphase entry rarely focus on the control of timing of mitotic events during embryo development, yet the control of the mitotic timing is of great importance for the correct developmental process (Krasinaska et al., 2011; Gharbi-Ayachi et al., 2010; Moshida et al., 2010). To unravel developmental aspects of cell cycle regulation we asked whether the reciprocal regulation between CDK1 and PP2A might serve as a mechanism specifying the timing of the first embryonic M-phase. Here, we show using Xenopus laevis onecell embryo cell-free extract that the balance between CDK1 and okadaic acid-sensitive phosphatases (especially PP2A) precisely controls, in coordination with the accumulation of cyclin, the timing of entry and exit from the first embryonic M-phase.

\section{Results}

\section{Timing of M-phase entry is robustly controlled in cell-free extract}

We tested here in cytoplasmic extracts whether RO 3360 (RO), a selective CDK1 inhibitor (Vassilev et al., 2006), or okadaic acid (OA), known to inhibit both PP2A and PP1 phosphatase (Felix et al., 1990), can modify the timing of M-phase entry. Before adding inhibitors, we checked the robustness of the control of timing of M-phase entry by adding to the extracts ethanol (solvent of RO) or DMSO (solvent of $\mathrm{OA}$ ) or the mixture of the two solvents in concentrations of $1.5 \%$. We also tested effects of higher concen- tration (up to $4 \%$ ) of these solvents to evaluate the upper limit not influencing the timing of the M-phase entry. Modifications of CDC27 migration in PAGE due to CDK1 phosphorylation (up-shift) were used as molecular markers of the M-phase entry. No difference was found in the timing of CDC27 phosphorylation, and thus in the timing of M-phase entry, when ethanol or DMSO or both were added to the extracts at the concentration of $1.5 \%$ (Fig. $1 \mathrm{~A})$, thus this concentration was used in subsequent experiments. We also confirmed that the mixture of $1.5 \%$ ethanol and DMSO did not change the timing of M-phase entry (Fig. 1B second row). Higher concentration of solvents (4\%) in the extracts inhibited their Mphase entry (Fig.1B, bottom row). In addition, we confirmed that the separate aliquots of the same extract incubated in separate tubes entered M-phase exactly at the same time in all tubes (data not shown). These results indicated that the M-phase entry is robustly controlled in cell-free extract and the timing of M-phase entry is not modified by the presence of ethanol, DMSO or mixture of both in concentration not exceeding $1.5 \%$.

Timing of M-phase entry is delayed by Inhibition of CDK1 and accelerated by inhibition of OA-sensitive phosphatases

$\mathrm{RO}$ or OA were added to the extract before M-phase entry. $\mathrm{RO}$ was added at 1, 2 and $10 \mu \mathrm{M}$ concentration (Vassilev et al., 2006), while OA at 1-2.5 $\mu \mathrm{M}$ concentration (Felix et al., 1990). We found that the inhibition of CDK1 or OA-sensitive phosphatases changed the timing of $\mathrm{M}$-phase entry in a dose-dependent manner. Inhibition of CDK1 using low ( 1 or $2 \mu \mathrm{M}$ ) doses of RO, delayed the mitotic entry by 4 and 20 minutes respectively as compared to the control. However, the addition of $10 \mu \mathrm{M}$ RO inhibited the M-phase entry for at least 32 min, at which point the experiment was terminated. Thus, we can't say with certainty if the M-phase was totally inhibited or severely (for $32 \mathrm{~min}$ ) delayed (Fig 2A). In contrast, the addition of 1 and $2.5 \mu \mathrm{M}$ of the $\mathrm{OA}$, which drastically inhibits both PP2A and PP1 (Felix et al., 1990), accelerated the $\mathrm{M}$-phase entry by 24 and 32 min respectively (Fig $2 B$ ). Thus, despite that the timing of M-phase entry is robustly controlled in the cell-free extracts it can be modified by manipulation of the activities of CDK1 or OA-sensitive phosphatases.

\section{Simultaneous partial inhibition of CDK1 and PP2A results in intermediate timing of M-phase entry}

Because CDK1 inhibition and OA-sensitive phosphateses inhibition have opposite effects on the timing of the M-phase entry, we used low concentrations of the drugs; 2 and $4 \mu \mathrm{M}$ of $\mathrm{RO}$ to partially inhibit CDK1 and $200 \mathrm{nM}$ OA to partially inhibit PP2A
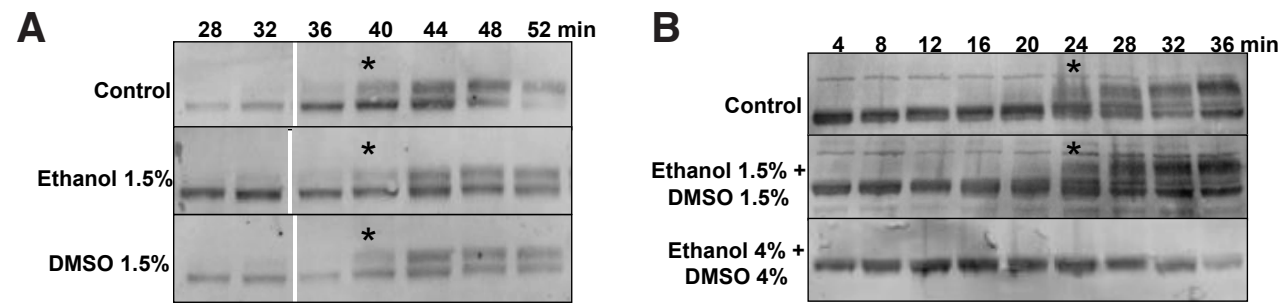

Fig. 1. Test of robustness of the control extract in regard to the timing of the $\mathrm{M}$ phase entry. (A) The cytoplasmic extract prepared 65 min post-activation, incubated at $21^{\circ} \mathrm{C}$ in the presence of $1.5 \%$ ethanol (the solvents of RO) and DMSO $1.5 \%$ (the solvent of $O A$ ) was sampled every 4 minutes. Samples were analyzed by $8 \%$ SDS-PAGE followed by CDC27 Western blot. (B) Another extract was prepared 60 min post-activation, incubated at $21^{\circ} \mathrm{C}$ in the presence of both 1.5\% ethanol and 1.5\% DMSO (second row). Note that the simultaneous presence of $1.5 \%$ ethanol and $1.5 \%$ DMSO does not change the timing of M-phase entry as compared to the control. The same extract was incubated with a high concentration of ethanol (4\%) and DMSO (4\%), which abolishes M-phase entry during 36 min incubation. This experiment shows that 1.5\% ethanol and $1.5 \%$ DMSO do not interfere with the timing of M-phase entry, while 4\% ethanol and 4\% DMSO do. Asterisk mark the time points estimated as the M-phase entry. 

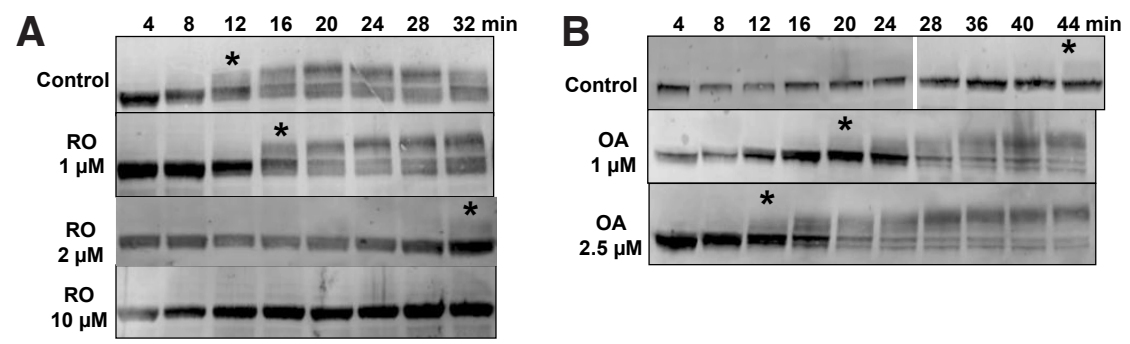

Fig. 2. The inhibition of CDK1 and okadaic acid (OA)-sensitive phosphatases separately changes the timing of M-phase entry in a dose-dependent manner in Xenopus laevis embryo cell-free extract. (A) The low-speed cytoplasmic extract was incubated at $21^{\circ} \mathrm{C}$ in the presence of three $(1,2$ or $10 \mu \mathrm{M})$ concentrations of $R O 3306$ (RO) and blotted for CDC27. The phosphorylated forms of CDC27 appearing at the M-phase entry due to phosphorylation by CDK1 were identified by delayed migration in SDS-PAGE. Note that increased concentrations of RO delay the M-phase entry in a dose-dependent manner. Asterisks indicate the time points when substantial shift in CDC27 migration was observed and which were assumed as the M-phase entry. No M-phase entry was observed during 32 min incubation in the $10 \mu \mathrm{M}$ $R O$ treated extract (bottom). (B) The extract was incubated at $21^{\circ} \mathrm{C}$ without drugs (control) and in the presence of two concentrations of the inhibitor of phosphatases okadaic acid (OA): $1 \mu \mathrm{M}$ or $2.5 \mu \mathrm{M}$. Note that increased concentrations of OA accelerate M-phase entry in a dose-dependent manner. Asterisks indicate the time when substantial shift in CDC27 migration was observed.

without substantial inhibition of PP1 (Felix et al., 1990). The presence of $2 \mu \mathrm{M}$ RO delayed the M-phase entry by 16 minutes, while $4 \mu \mathrm{M}$ RO delayed it by 20 minutes (Fig. 3), however, the presence of $200 \mathrm{nM}$ OA did not induce any change in the time of M-phase entry (Fig. 3, fourth row). Our choice to work with either $200 \mathrm{nM}$ or lower concentration of $\mathrm{OA}$, was determined by the fact that these concentrations of OA are known to inhibit partially PP2A, and not PP1. These low concentrations of OA did not modify the timing of M-phase entry, which indicates that partial inhibition of PP2Aactivity is efficiently neutralized in the cell-free extract. However, when 200 $\mathrm{nM}$ OA and $2 \mu \mathrm{M} \mathrm{RO}$ were added simultaneously, there was $4 \mathrm{~min}$ delay in the M-phase entry in comparison to the control extracts, and $12 \mathrm{~min}$ acceleration of the M-phase entry in comparison to the extract supplemented with $2 \mu \mathrm{M}$ RO alone (Fig. 3, fifth row). Moreover, when $200 \mathrm{nM} O A$ and $4 \mu \mathrm{M}$ RO were added simultaneously, the M-phase entry was delayed by $8 \mathrm{~min}$ (Fig. 3, sixth row) in comparison to the control sample (Fig. 3, first row) and accelerated by $12 \mathrm{~min}$ in comparison to the extract supplemented with $4 \mu \mathrm{M} R O$ alone (Fig. 3, third row). As expected, the $2.5 \mu \mathrm{M}$ OA added to the extract accelerated M-phase entry by 8 minutes in comparison to the control, confirming that OA used in the experiment was indeed active (Fig. 3, bottom row). These results summarised in Table 1

TABLE 1 TIMING OF M-PHASE ENTRY AS ESTIMATED BY THE SHIFT OF
CDC27 ON THE WESTERN BLOT SHOWN IN FIG. 3

\begin{tabular}{lc} 
Experimental variant & M-phase entry \\
\hline Control & $20 \mathrm{~min}$ \\
RO $2 \mu \mathrm{M}$ & $36 \mathrm{~min}$ \\
$\mathrm{RO} 4 \mu \mathrm{M}$ & $40 \mathrm{~min}$ \\
$\mathrm{OA} 200 \mathrm{nM}$ & $20 \mathrm{~min}$ \\
OA $200 \mathrm{nM}+\mathrm{RO} 2 \mu \mathrm{M}$ & $24 \mathrm{~min}$ \\
OA $200 \mathrm{nM}+\mathrm{RO} 4 \mu \mathrm{M}$ & $28 \mathrm{~min}$ \\
OA $2.5 \mu \mathrm{M}$ & $12 \mathrm{~min}$ \\
\hline
\end{tabular}

show that the simultaneous partial inhibition of CDK1 and PP2A results in an intermediate timing of M-phase entry as compared to the control and CDK1 or PP2A inhibition alone. This, in turn, suggests that CDK1 inhibition equilibrates the down regulation of PP2A and vice versa. Thus, a balance between the activities of CDK1 and PP2A controls not only the induction, but also specifies the timing of M-phase entry.

Down regulation of CDK1 and PP2A delays M-phase exit and prolongs $M$-phase duration in a dose-dependent manner

We next asked the question whether partial inhibition of CDK1 and PP2A can modify the timing of the M-phase exit. We performed two sets of experiments; in one set we supplemented extracts either with $\mathrm{OA}$ or $\mathrm{RO}$ and in another set we added $\mathrm{OA}$ and RO together. We than followed the biochemical changes for the period of $72 \mathrm{~min}$, which in control extracts includes the exit from M-phase. In order to be sure that our OA treatment does not diminish PP1 activity we further diminished the concentration of OA to $50 \mathrm{nM}$. At this concentration the PP1 is still active and PP2A is inhibited to lower degree than in the presence of 200 nM OA (Felix et al., 1990). To study the dynamics of phosphorylation and dephosphorylation of CDK1 substrates we performed Western blot analysis of two different substrates with different dynamic of phosphorylation: fast phosphorylated MCM4

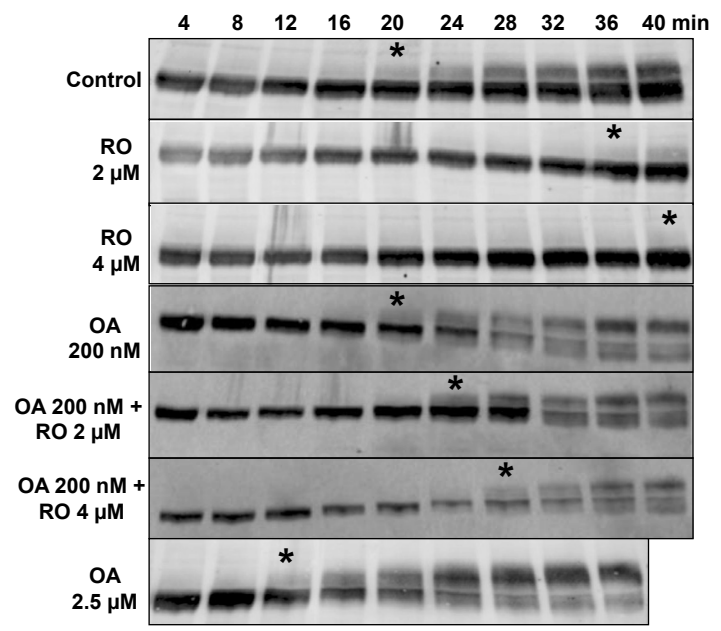

Fig. 3. The combination of CDK1 and PP2A inhibitors results in the Mphase entry timing intermediate between control and extracts treated with R03306 (RO) or okadaic acid (OA) alone. (A) The simultaneous partial inhibition of CDK1 and PP2A modifies the timing of M-phase entry in a dose-dependent manner as compared to CDK1 and PP2A inhibition alone. Cell-free extract was incubated separately with 2 or $4 \mu M$ RO 3306 (RO) or with $200 \mathrm{nM}$ (fourth row) or $2.5 \mu \mathrm{M}$ (bottom row) okadaic acid (OA). In parallel experiments both $R O$ and OA were added simultaneously. Note that $200 \mu \mathrm{M}$ OA has no effect on the timing of M-phase entry when compared to control (first row), but simultaneous presence of 200 $\mathrm{MM}$ OA and 2 or4 $\mu M R O$ results in dose-dependent delay of the M-phase entry (row 5 and 6). The bottom row shows that $2.5 \mu \mathrm{M} \mathrm{OA}$ is fully active and accelerates $M$-phase in comparison to the control. 
A

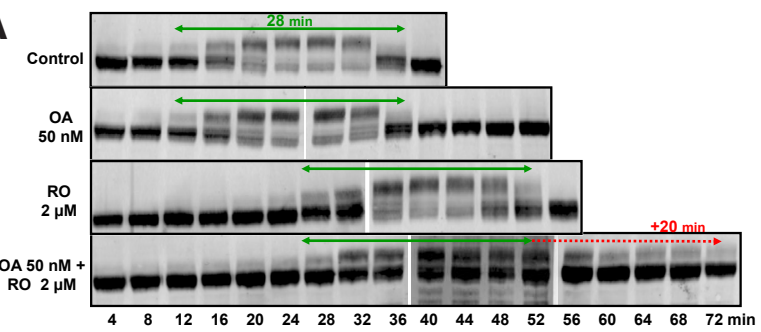

C

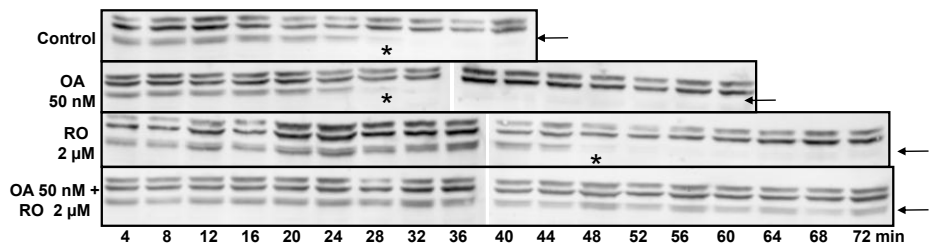

B
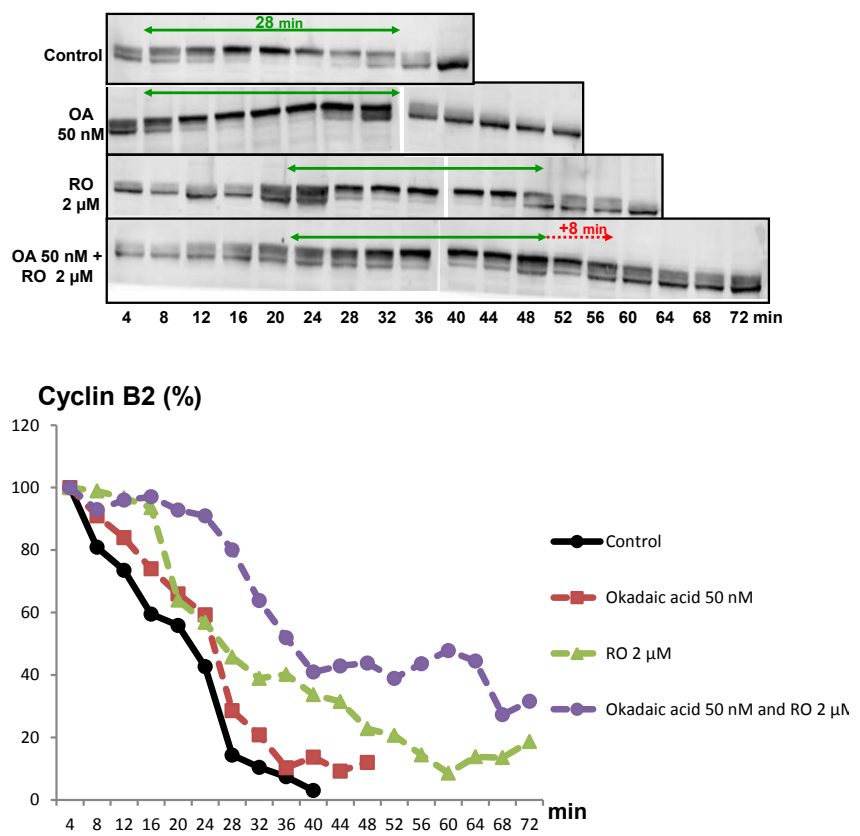

Fig. 4. The simultaneous inhibition of CDK1 and PP2A modifies the timing of M-phase exit and duration. Cell-free extract was incubated without drugs (control, the first row) or with: $50 \mathrm{nM} \mathrm{OA}$ (second row), $2 \mu \mathrm{M} \mathrm{RO}$ (third row), both $50 \mathrm{nM} O \mathrm{~A}$ and $2 \mu \mathrm{M}$ RO (bottom row). (A) Western blots with anti-CDC27 antibody. Double-headed arrows show the estimated duration of the period when CDC27 is up-shifted indicating the duration of the M-phase. The prolongation of the period of CDC27 phosphorylation is marked with pointed arrow. (B) Western blot with anti-MCM4 antibody. The double-headed arrows show the duration of the period when up-shift of MCM4 dominates. Note that MCM4 shows the up-shift earlier than CDC27. The prolongation of MCM4 dephosphorylation states (including only the period when the uppermost band of MCM4 was present) are marked with pointed arrows. Following this period MCM4 is still not fully dephosphorylated till the end of the experiment at 72 min. (C) Western blot with anti-cyclin B2 antibody. Asterisks show the time when cyclin B2 massive degradation begins. Note that in the presence of both $50 \mathrm{nM} O A$ and $2 \mu \mathrm{M} R O$ (bottom row) cyclin $B$ is not degraded till the end of experiment at $72 \mathrm{~min}$. Arrows on the right show the position of cyclin B2. Note that the unspecific double band of slightly higher MW than cyclin B2 serves as a convenient loading control for cyclin B2. (D) Quantification of the cyclin B2 Western blot from Fig. 4C.

and slow phosphorylated CDC27 (see below). We found out that in 50 nM OA-treated extracts the CDC27 was phosphorylated and dephosphorylated with exactly the same dynamics like in the control (Fig. 4A, first and second row). Treatment of the extracts with $2 \mu \mathrm{M}$ $\mathrm{RO}$ alone delayed CDC27 phosphorylation, but the duration of the $M$-phase was exactly the same as in the control and OA-treated extracts (Fig. 4A, third row). Addition of $2 \mu \mathrm{M}$ RO and $50 \mathrm{nM} O A$ together did not modify the timing of M-phase entry. This result and the result of previous experiment (showing that $2 \mu \mathrm{M} \mathrm{RO}$ and $200 \mathrm{nM}$ OA added together delayed timing of M-phase entry; see Fig. 3, fifth row) demonstrate existence of a threshold in PP2A inhibition above which the timing of M-phase changes. However, the timing of $\mathrm{M}$-phase exit and the M-phase duration changed drastically when $2 \mu \mathrm{M} \mathrm{RO}$ and $50 \mathrm{nM}$ OA were used together (Fig. $4 \mathrm{~A}$, compare the third and fourth row). During the M-phase exit in the presence of two drugs, dephosphorylation of CDC27 was dramatically slowed down and phosphorylated CDC27 was present until the end of experiment (72 minutes) prolonging the M-phase duration for about 20 min (Fig. 4A fourth row). For clarity, these results are summarized in Table 2 . In parallel, we analysed the phosphorylation pattern of MCM4 that is phosphorylated very early upon M-phase entry (Chesnel et al., 2005). As expected, MCM4 phosphorylation occurred faster than CDC27 during the M-phase entry, but it was also dephosphorylated faster upon M-phase exit (Fig. 4B) resulting in a constant M-phase duration. Thus, the combination of $2 \mu \mathrm{M}$ RO and $50 \mathrm{nM}$ OA did not modify the timing of M-phase entry, but delayed the M-phase exit and slowed down the dynamics of phosphorylation and dephosphorylation of the two CDK1 substrates, while 50nM OA added alone had no effect on the dynamics of these processes. Thus, the regulation of the timing of M-phase exit is even more sensitive to modifications of CDK1/PP2A equilibrium than the timing of M-phase entry and the balance between activities of CDK1 and PP2A plays a major role in regulation of both $\mathrm{M}$-phase entry and exit, and therefore in the progression of M-phase.

\section{Delayed M-phase exit implies modified dynamics of cyclin B2 degradation}

The timings of M-phase entry and exit are directly regulated by the dynamics of cyclin B metabolism (Murray and Kirschner, 1989;

TABLE 2

\section{TIMING OF M-PHASE ENTRY AND EXIT AS ESTIMATED BY THE SHIFT OF CDC27 ON THE WESTERN BLOT SHOWN IN FIG. 4}

\begin{tabular}{lccc} 
Experimental variant & M-phase entry & M-phase exit & M-phase duration \\
\hline Control & $12 \mathrm{~min}$ & $36 \mathrm{~min}$ & $24 \mathrm{~min}$ \\
OA $50 \mathrm{nM}$ & $12 \mathrm{~min}$ & $36 \mathrm{~min}$ & $24 \mathrm{~min}$ \\
RO $2 \mu \mathrm{M}$ & $28 \mathrm{~min}$ & $52 \mathrm{~min}$ & $24 \mathrm{~min}$ \\
OA $50 \mathrm{nM}+\mathrm{RO} 2 \mu \mathrm{M}$ & $28 \mathrm{~min}$ & $72 \mathrm{~min}$ & $44 \mathrm{~min}$ \\
\hline
\end{tabular}


Murray et al., 1989; Glotzer et al., 1991). In order to assess cyclin $B$ dynamics and its involvement in modifications of the M-phase entry and exit, we analysed cyclin B dynamics in the experiment described in previous paragraph (Fig. 4C; the time points of cyclin B2 degradation are labelled with asterisks). We found that the dynamics of cyclin B2 degradation were similar in control and in $50 \mathrm{nM}$ OA-treated extracts (Fig. 4C). The presence of $2 \mu \mathrm{M}$ RO alone in the extracts significantly delayed cyclin B2 degradation (Fig. 4C, third row; compare with graph in Fig. 4D). Most importantly, upon simultaneous presence of $2 \mu \mathrm{M}$ RO and $50 \mathrm{nM}$ OA cyclin B2 diminished but did not rich the point of total degradation until the end of the experiment (at the time point 72 min; Fig. 4C, fourth row and the graph at Fig. 4D).

In order to check more precisely how the modifications of PP2A influence CDK1 activity we used two (100 and 200nM) concentrations of $\mathrm{OA}$ in conjunction with lower RO concentration (1 $\mu \mathrm{M})$. $100 \mathrm{nM}$ OA applied alone did not change the parameters of either M-phase entry or exit (Fig. 5A, second row). 200 nM OA alone did not modify the timing of the M-phase entry but slightly delayed the M-phase exit, and thus prolonged by 4 minutes the duration of the M-phase (Fig. 5A, third row). $1 \mu \mathrm{M} \mathrm{RO}$ alone delayed the M-phase entry (as shown previously on Fig. 2A), but did not prolong the duration of the M-phase (Fig. 5A, fourth row). In contrast, $1 \mu \mathrm{M}$ $\mathrm{RO}$ added simultaneously with $100 \mathrm{nM}$ OA prolonged the duration of the M-phase by $8 \mathrm{~min}$ (Fig. 5A, fifth row). When $200 \mathrm{nM} \mathrm{OA}$ was added together with $1 \mu \mathrm{M} \mathrm{RO}$, the duration of the M-phase was 16 min longer (Fig. 5A, bottom row). These results are summarized in Table 3. MCM4 Western blotting confirmed the same tendencies (Fig. 5 A,B). Cyclin B2 Western blotting confirmed that degradation of this protein is also delayed when CDK1 substrates phosphorylation is prolonged (Fig. 5 C,D). The delay in cyclin B2 degradation significantly increased when $1 \mu \mathrm{M}$ RO and $200 \mathrm{nM}$ $\mathrm{OA}$ were simultaneously present in the extract and correlated with the highest delay in the M-phase exit (Fig. 5 C,D). Interestingly, the dynamics of cyclin B2 accumulation was unchanged in all experimental groups and we detected the maximum levels of
A

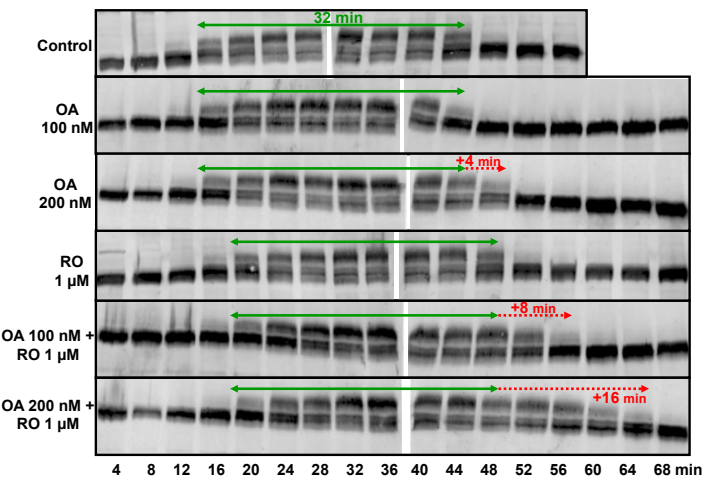

B

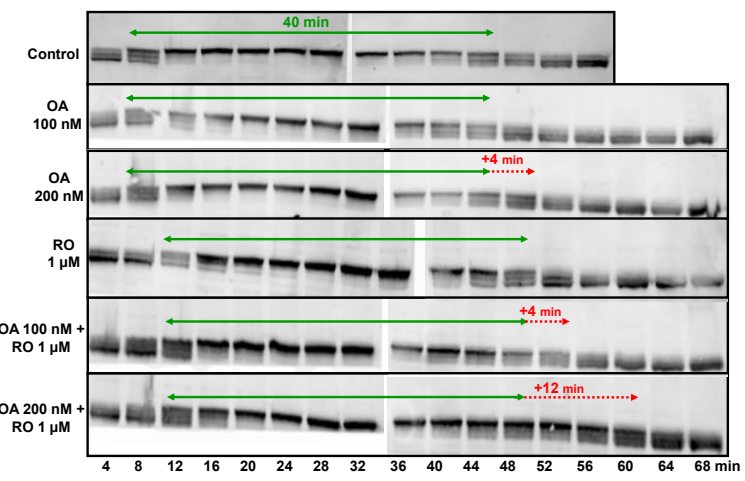

D

C

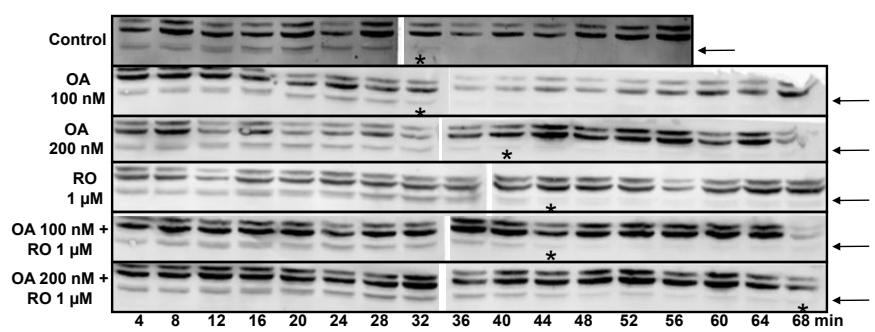

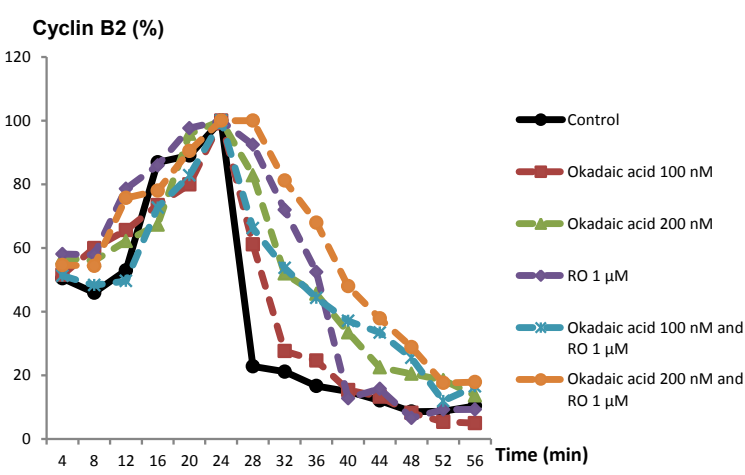

E

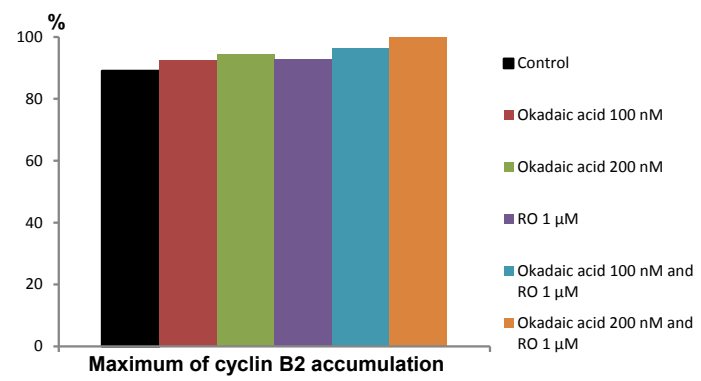

Fig. 5.The simultaneous inhibition of CDK1 and PP2A modifies the timing of M-phase exit and M-phase duration in a dose-dependent manner. $100 \mathrm{nM}$ or $200 \mathrm{nM}$ OA or $1 \mu \mathrm{M}$ $R O$ were separately added to the extracts (rows two, three and four). (A) Samples were analyzed by $8 \%$ SDS-PAGE followed by Western blotting with anti-CDC27. (B) Western blot with anti-MCM4 (C) Western blot with anti-cyclin B2 antibody. (D) Quantification of the cyclin B2 Western blot. Note that the dynamics of cyclin B2 accumulation do not vary significantly in different combination of drugs, while the dynamics of cyclin B2 degradation is significantly different (for details see the Result section). (E) The comparison of the maximum levels of cyclin B2 at 24 min incubation in the presence of the different combinations of inhibitors shows very small differences. Double-headed arrows show the duration of maximum up-shift of CDC27 (A) and MCM4 (B). The prolongation of the persistence of the phosphorylated forms of these proteins is marked with pointed arrows. 
TABLE 3

TIMING OF M-PHASE ENTRY AND EXIT AS ESTIMATED BY THE SHIFT OF CDC27 ON THE WESTERN BLOT SHOWN IN FIG. 5

\begin{tabular}{lccc} 
Experimental variant & M-phase entry & M-phase exit & M-phase duration \\
\hline Control & $16 \mathrm{~min}$ & $44 \mathrm{~min}$ & $28 \mathrm{~min}$ \\
OA $100 \mathrm{nM}$ & $16 \mathrm{~min}$ & $44 \mathrm{~min}$ & $28 \mathrm{~min}$ \\
OA $200 \mathrm{nM}$ & $16 \mathrm{~min}$ & $48 \mathrm{~min}$ & $32 \mathrm{~min}$ \\
RO $1 \mu \mathrm{M}$ & $20 \mathrm{~min}$ & $48 \mathrm{~min}$ & $28 \mathrm{~min}$ \\
OA $100 \mathrm{nM}+$ RO $1 \mu \mathrm{M}$ & $20 \mathrm{~min}$ & $56 \mathrm{~min}$ & $36 \mathrm{~min}$ \\
OA 200 nM + RO $1 \mu \mathrm{M}$ & $20 \mathrm{~min}$ & $64 \mathrm{~min}$ & $44 \mathrm{~min}$ \\
\hline
\end{tabular}

cyclin B2 at 24 min of incubation in all samples (Fig. 5 D,E). These results indicate that although the $\mathrm{OA}$ and $\mathrm{RO}$ treatments do not significantly modify the dynamics of cyclin B2 accumulation, they significantly modify the dynamics of its degradation. Thus, judging by differential effects of low doses of $\mathrm{RO}$ and $\mathrm{OA}$, the regulation of the timing of M-phase exit is even more sensitive to the equilibrium between CDK1 and PP2A activities than the timing of the M-phase entry. Moreover, regulation of the timing of the M-phase exit relies on the control of the dynamics of cyclin B degradation.

\section{Discussion}

The major role of cyclin accumulation in determining the timing of the M-phase entry in Xenopus laevis one-cell embryo has been established over 20 years ago (Murray \& Kirschner; 1989; Murray, Glotzer \& Kirschner, 1989). Yet, it remains completely unknown how such simple mechanism can precisely tune temporal parameters of the M-phase. The coordination between the timing of cell divisions and the genetic program of development is crucial for normal embryo development. Therefore, it must be controlled with extreme accuracy. In somatic cells, a sophisticated system of checkpoints monitors when mitosis takes place and how fast it progresses (Rieder et al., 1995). Thus, the simple mechanism based on the accumulation/degradation of a single molecule such as cyclin (Murray \& Kirschner; 1989; Murray, Glotzer \& Kirschner, 1989) might not be fully adequate to account for the precise control of cell division timing in the early embryo. Since CDK1 activity itself is necessary to trigger CDC25/CDK1 activating loop, the dynamics of the initial, slow phase of CDK1 activation should influence the ability to activate CDC25 and consequently the activation of the feedback loop. Here we tested this hypothesis using a pharmacological approach allowing moderate inhibition of CDK1.

We found that the timing of the M-phase entry does not depend exclusively on the level of cyclin accumulation, but can be modulated by the initial activity of both CDK1 and OA-sensitive phosphatases, mostly PP2A (Felix et al., 1990). It was shown by Krasinska et al., (2011) that the equilibrium between CDK1 and PP2A plays a key role in induction of the M-phase entry. However, these authors used relatively high concentration of OA $(1 \mu \mathrm{M})$, which totally inhibits PP2A. Thus, they could observe only the all-or-none response in respect of $\mathrm{M}$-phase induction. The partial inhibition of CDK1 or PP2A in Xenopus embryo extracts modifies the timing of M-phase entry in a dose-dependent manner: the M-phase entry is delayed in extracts treated with CDK1 inhibitor $\mathrm{RO}$ and accelerated upon treatment with OA. This suggests an important role of the initial level of CDK1 activation preceding the CDC25 activation and the triggering of the activation loop responsible for the massive activation of CDK1. It is well known that the inhibition of OA-sensitive phosphatases PP1 and PP2A results in activation of CDK1 even in interphase extracts (Félix et al., 1990). Here we show that partial inactivation of PP2A (low doses of OA; 50-200 nM), does not modify the timing of M-phase entry suggesting that the moderate diminution in PP2A activity is compensated in the extract. The simultaneous partial inhibition of CDK1 and PP2A (with low doses of RO and OA), which resulted in an intermediate timing of M-phase entry shows the importance of the interplay between these two enzymes in regulation of the mitotic timing. Our results strongly suggest that besides the simple accumulation of cyclin to a threshold level, the CDK1 and PP2A cooperation plays an important role in the tuning of the time of $\mathrm{M}$ phase entry. The key information here is that while a very subtle inhibition of PP2A alone has no effect on the M-phase timing, the simultaneous subtle inhibition of PP2A and CDK1 has profound effect on M-phase timing. This argues for the importance of the tuning of these two activities for the correct timing of the M-phase entry in the embryo.

The delayed M-phase entry provoked by low doses of RO inhibiting the initial activity of CDK1 takes place when the level of cyclins $B$ is higher than that in control extract upon M-phase entry. Conversely, in the presence of low concentrations of $O A$, the Mphase entry takes place in spite of the low level of cyclin B2. Thus, we conclude that cyclin B accumulation determines only a window of opportunity in which the M-phase entry may take place, while the exact timing of M-phase entry is specified by the activation of low levels of CDK1 in coordination with the inactivation of PP2A. In addition, the lack of effect of low doses of OA alone on the timing of M-phase entry suggests that PP2A and CDK1 remain in constant reciprocal control during the initial phases of the M-phase.

In the interphase, during the cyclin accumulation phase, the type 2A phosphatase binds to CDC25C (Margolis et al., 2006). This interaction controls phosphorylation of critical inhibitory sites on CDC25C inducing its inhibition. However, at the end of the interphase the very first CDK1 molecules trigger PP2A inhibition via Greatwall kinase activation (Vigneron et al., 2009) and at the same time promotes CDC25 activation by two means: through its active phosphorylation by CDK1 and through the preservation of this phosphorylation due to PP2A inhibition. Thus, the appearance of the very first CDK1 active molecules plays a crucial role in the complex process triggering the M-phase entry. The presence of low doses of RO (this paper) clearly delays this moment. Thus, we show here that the precise timing of this critical event indeed depends on the initial activity of CDK1, and in addition that it is controlled by a dialog between CDK1 and PP2A.

We also showed here that the simultaneous partial inhibition of CDK1 and PP2A substantially modifies the timing of the exit and thus the duration of the M-phase. Any change of this equilibrium results in prolongation or shortening of the M-phase. Moreover, the delayed M-phase exit under combined treatment with RO and OA drastically slowed down the rate of cyclin B degradation. Therefore, the modified timing of M-phase exit involves most probably lower level of the ubiquitin ligase APC/C activation triggering cyclin $B$ degradation, which results from a modified balance between CDK1-dependent phosphorylations and PP2A-dependent dephosphorylations of APC/C components or regulators. Thus, the timing of both M-phase entry and exit seems to be determined by a subtle equilibrium between CDK1, PP2A and by the threshold of 
cyclin level. The threshold of cyclin B sufficient to trigger M-phase entry can be apparently compensated by modification of the initial activity of CDK1 and PP2A. Such flexibility may allow the embryo to better coordinate the cell cycle and developmental programs and adjust the timing of mitotic divisions during early development. We have shown previously that the duration of the first two embryonic M-phases in Xenopus laevis correlates with the level of cyclin B accumulation (Chesnel et al., 2005). Our current results show that it also depends on the degree of CDK1 activation tightly linked to PP2A inactivation. Thus, M-phase duration is regulated by the interplay between the level of cyclin $B$ accumulation and the balance between activities of CDK1 and PP2A. The clear differences in the sensitivity of the control of M-phase entry and exit to the modified balance between CDK1 and PP2A activities shown in our study suggest that the embryo may regulate the cell cycle progression not only at the level of M-phase entry, but also through controlled timing of M-phase duration. The existence of similar embryonic strategy has been shown by us before in respect to the regulation of the timing of the first and second mitotic division both in Xenopus and mouse embryos (Chesnel et al., 2005; Ciemerych et al., 1999; Sikora-Polaczek et al., 2006; reviewed in Kubiak et al., 2008a and in Kubiak et al., 2008b).

\section{Material and Methods}

\section{Egg collection and activation}

Xenopus laevis females were purchased from NASCO (Fort Atkinson, WI, USA). Females were injected subcutaneously with Human Chorionic Gonadotropin HCG (500 IU per female) and kept overnight at $21^{\circ} \mathrm{C}$ in 110 $\mathrm{mM} \mathrm{NaCl}$. Cell-free extracts were prepared as previously described by Murray (1991) using modifications described by Chesnel and colleagues (2005). Metaphase-arrested Xenopus eggs, collected from overnight spawning after HCG injection, were dejellied with $2 \% \mathrm{~L}$-cysteine $\mathrm{pH} 7.81$ in XB buffer (100 mM KCl, $1 \mathrm{mM} \mathrm{MgCl}, 50 \mathrm{mM} \mathrm{CaCl} 2,10 \mathrm{mM}$ HEPES, and 50 $\mathrm{mM}$ sucrose $\mathrm{pH}$ 7.6). Dejellied eggs were washed in XB buffer, activated with $0.5 \mathrm{mg} / \mathrm{ml}$ of calcium ionophore A23187 until cortical contraction was observed, and then extensively washed in XB.

\section{Cell free extracts}

Cytoplasmic extracts from calcium ionophore-activated embryos before the first embryonic mitosis were prepared according to Murray (1991). Embryos were cultured at $21^{\circ} \mathrm{C}$ in XB Buffer for $60-70$ minutes post-activation, i.e. till the late first interphase as described before (Chesnel et al., 2005). Embryos were then transferred into $5 \mathrm{~mL}$ ultra-clear ${ }^{\mathrm{TM}}$ centrifuge tubes (Beckman Coulter, Roissy, France) and incubated at $4^{\circ} \mathrm{C}$ in $0.5 \mathrm{~mL}$ of $\mathrm{XB}$ buffer containing protease inhibitors $(10 \mu \mathrm{g} / \mathrm{ml}$ of each aprotinin, leupeptin, pepstatin, chymostatin, and $0.1 \mathrm{mM} \mathrm{AEBSF}$ ) and $25 \mathrm{mg} / \mathrm{ml}$ of cytochalasin D. Subsequently, embryos were subjected to three consecutive centrifugations: first short spin to remove $\mathrm{XB}$ excess and pack the embryos in the tube, second $10.000 \mathrm{~g}$ spin at $4^{\circ} \mathrm{C}$ for 10 minutes to separate the embryo fractions, and final $10.000 \mathrm{~g}$ clarification spin of the supernatant at $4^{\circ} \mathrm{C}$ for 10 minutes. The supernatant was then incubated at $21^{\circ} \mathrm{C}$. Every 4 minutes, aliquots were taken out and directly mixed with Laemmli buffer for Western blot analyses (Laemmli et al., 1970). All aliquots were stored at $-80^{\circ} \mathrm{C}$.

\section{Drugs}

RO 3360 (RO), a selective CDK1 inhibitor (Vassilev et al., 2006) was purchased from Merck Millipore. It was used in 1, 2, 4 and $10 \mu \mathrm{M}$ concentrations. The stock solution was dissolved in ethanol. Okadaic acid (OA), from Molecular Probes, known to inhibit both PP2A and PP1 phosphatase in high concentrations of 1-2.5 $\mu \mathrm{M}$ and PP2A in low concentrations of 50-200 nM (Felix et al., 1990) was purchased from Sigma. The stock solution was dissolved in DMSO. We used the low doses of OA (200, 100 and $50 \mathrm{nM})$ in the majority of our experiments because these dosages do not change the timing of M-phase entry when applied alone and according to the previous study they partially inhibit PP2A (Felix et al., 1990).

\section{Western blot and antibodies}

Protein extracts were subjected to $8 \%$ or $12.5 \%$ sodium dodecyl sulfate polyacrylamide gel electrophoresis (SDS-PAGE) (Laemmli et al., 1970). Separated proteins were transferred to nitrocellulose membranes (Hybond $\mathrm{C}$, Amersham Biosciences). Membranes were blocked with PBS containing $5 \%$ non-fat dry milk and $0.1 \%$ Tween 20 and probed with primary antibodies against Xenopus laevis CDC27, cyclin B2 (gift from T. Lorca and A. Castro) and MCM4 (gift from M. Mechali). Antigen-antibody complexes were visualized using alkaline phosphatase-conjugated anti-rabbit antibodies with detection by chemifluorescence using the ECF Reagent (Amersham Biosciences). Signal quantification was performed using ImageQuant 5.2 software (Amersham Biosciences). In most cases (Figs. 1A, 2B, 4 A,B,C, $5 A, B, C)$ the alignment of two or three separate Western blot membranes was used throughout this paper to show the time sequence of biochemical events. In all these cases the membranes were clearly separated by a blank space to highlight the fact that the aligned parts belong to different blots.

\section{Acknowledgements}

While this work was carried out MED was supported by Lebanese Ministry of Education. We are grateful to Thierry Lorca and Anna Castro (CRBM, Montpellier, France) for providing us antibodies against CDC27, CDC25C and cyclin B2.

\section{References}

CHESNEL F, VIGNAUX F, RICHARD-PARPAILLON L, HUGUET A, KUBIAK JZ. (2005). Differences in regulation of the first two M-phases in Xenopus laevis embryo cell-free extracts. Dev Biol 285: 358-375.

CHESNEL F, BAZILE F, PASCAL A, KUBIAK JZ. (2006). Cyclin B dissociation from CDK1 precedes its degradation upon MPF inactivation in mitotic extracts of Xenopus laevis embryos. Cell Cycle 5: 1687-1698.

CHESNEL F, BAZILE F, PASCALA, KUBIAK JZ. (2007). Cyclin B2/cyclin-dependent kinase1 dissociation precedes CDK1 Thr-161 dephosphorylation upon M-phase promoting factor inactivation in Xenopus laevis cell-free extract. Int J Dev Biol 51: 297-305.

CIEMERYCH MA, MARO B, KUBIAK JZ. (1999). Control of duration of the first two mitoses in a mouse embryo. Zygote 7: 293-300.

FELIX MA, PINES J, HUNT T, KARSENTI E. (1989). Temporal regulation of cdc2 mitotic kinase activity and cyclin degradation in cell-free extracts of Xenopus eggs. J Cell Sci Suppl 12: 99-116.

FISHER D, KRASINSKA L, COUDREUSE D and NOVÁK B(2012). Phosphorylation network dynamics in the control of cell cycle transitions. J Cell Sci 125: 4703-4711.

GHARBI-AYACHI A, LABBÉ JC, BURGESS A, VIGNERON S, STRUB JM, BRIOUDES E, VAN-DORSSELAER A, CASTRO A, LORCA T. (2010). The substrate of Greatwall kinase, Arpp19, controls mitosis by inhibiting protein phosphatase 2A. Science 330: 1673-1677.

GAUTIER J, SOLOMON MJ, BOOHER RN, BAZAN JF, KIRSCHNER MW. (1991). Cdc25 is a specific tyrosine phosphatase that directly activates p34cdc2. Cell 67: 197-211.

GLOTZER M, MURRAY AW, KIRSCHNER MW. (1991). Cyclin is degraded by the ubiquitin pathway. Nature 349: 132-138.

GOULD KL, NURSE P. (1989). Tyrosine phosphorylation of the fission yeast cdc2+ protein kinase regulates entry into mitosis. Nature 342: 39-45.

HACCARD O, JESSUS C. (2011). Greatwall kinase, ARPP-19 and protein phosphatase 2A: shifting the mitosis paradigm. Results Probl Cell Differ 53: 219-234.

KRASINSKAL, DOMINGO-SANANESMR, KAPUYO, PARISISN, HARKERB, MOORHEAD G, ROSSIGNOL M, NOVÁK B, FISHER D. (2012). Protein phosphatase 2A controls the order and dynamics of cell-cycle transitions. Mol Cell 44: 437-450.

KUBIAK JZ, CIEMERYCH MA, HUPALOWSKAA, SIKORA-POLACZEKM, POLANSKI Z. (2008a). On the transition from the meiotic to mitotic cell cycle during early mouse development. Int J Dev Biol 52: 201-217. 
KUBIAK JZ, CHESNELF, RICHARD-PARPAILLON L, BAZILE F, PASCALA, POLANSKI Z, SIKORA-POLACZEK M, MACIEJEWSKA Z, CIEMERYCH MA. (2008b). Temporal regulation of the first mitosis in Xenopus and mouse embryos. Mol Cell Endocrinol 282: 63-69.

KUMAGAI A, DUNPHY WG. (1991). The cdc25 protein controls tyrosine dephosphorylation of the cdc2 protein in a cell-free system. Cell 64: 903-914.

LAEMMLI UK. (1970). Cleavage of structural proteins during the assembly of the head of bacteriophage T4. Nature 227: 680-685.

LUNDGREN K, WALWORTH N, BOOHER R, DEMBSKI M, KIRSCHNER M, BEACH D. (1991). mik1 and wee1 cooperate in the inhibitory tyrosine phosphorylation of cdc2. Cell 64: 1111-1122.

MARGOLIS SS, PERRY JA, FORESTER CM, NUTT LK, GUO Y, JARDIM MJ, THOMENIUS MJ, FREEL CD, DARBANDI R, AHN JH, ARROYO JD, WANG XF, SHENOLIKAR S, NAIRN AC, DUNPHY WG, HAHN WC, VIRSHUP DM, KORNBLUTH S. (2006). Role for the PP2A/B56delta phosphatase in regulating 14-3-3 release from Cdc25 to control mitosis. Cell 127: 759-773.

MOCHIDA S, IKEO S, GANNON J, HUNT T. (2009). Regulated activity of PP2A-B55 delta is crucial for controlling entry into and exit from mitosis in Xenopus egg extracts. EMBO J 28: 2777-2785.

MOCHIDA S, MASLEN SL, SKEHEL M, HUNT T. (2010). Greatwall phosphorylates an inhibitor of protein phosphatase $2 \mathrm{~A}$ that is essential for mitosis. Science 330 : 1670-1673.

MURRAY AW, KIRSCHNER MW. (1989). The role of cyclin synthesis and degradation in the control of maturation promoting factor activity. Nature 339: 275-280.

MURRAY AW, SOLOMON MJ, KIRSCHNER MW. (1989). The role of cyclin synthesis and degradation in the control of maturation promoting factor activity. Nature 339: $280-286$
MURRAY AW. (1991). Cell cycle extracts. Methods Cell Biol 36: 581-605.

NISHIYAMA A, TACHIBANA K, IGARASHI Y, YASUDA H, TANAHASHI N, TANAKA K, OHSUMI K, KISHIMOTO T. (2000). A nonproteolytic function of the proteasome is required for the dissociation of $\mathrm{Cdc} 2$ and cyclin $\mathrm{B}$ at the end of $\mathrm{M}$ phase. Genes Dev 14: 2344-2357.

PARKER LL, ATHERTON-FESSLER S, PIWNICA-WORMS H. (1992). p107wee1 is a dual-specificity kinase that phosphorylates p34cdc2 on tyrosine 15. Proc Nat Acad Sci USA 89: 2917-2921.

RIEDER CL, COLE RW, KHODJAKOV A, SLUDER G. (1995). The checkpoint delaying anaphase in response to chromosome monoorientation is mediated by an inhibitory signal produced by unattached kinetochores. J Cell Biol 130: 941-948.

SEBASTIAN B, KAKIZUKA A, HUNTER T. (1993). A phosphatase threshold sets the level of Cdk1 activity in early mitosis in budding yeast. Proc Natl Acad Sci USA 90: 3521-3524.

SIKORA-POLACZEK M, HUPALOWSKAA, POLANSKI Z, KUBIAK JZ, CIEMERYCH MA. (2006). The first mitosis of the mouse embryo is prolonged by transitional metaphase arrest. Biol Reprod 74: 734-743.

VASSILEV LT, TOVAR C, CHEN S, KNEZEVIC D, ZHAO X, SUN H, HEIMBROOK DC, CHEN L. (2006). Selective small-molecule inhibitor reveals critical mitotic functions of human CDK1. Proc Natl Acad Sci USA 103: 10660-10665.

VIGNERON S, BRIOUDESE, BURGESSA, LABBÉ JC, LORCAT, CASTROA. (2009) Greatwall maintains mitosis through regulation of PP2A. EMBO J 28: 2786-2793.

WU JQ, GUO JY, TANG W, YANG CS, FREELCD, CHENC, NAIRNAC, KORNBLUTH S. (2009). PP1-mediated dephosphorylation of phosphoproteins at mitotic exit is controlled by inhibitor-1 and PP1 phosphorylation. Nat Cell Biol 11: 644-651. 


\section{Further Related Reading, published previously in the Int. J. Dev. Biol.}

Sexual dimorphism of AMH, DMRT1 and RSPO1 localization in the developing gonads of six anuran species

Rafal P. Piprek, Anna Pecio, Katarzyna Laskowska-Kaszub,Jacek Z. Kubiak and Jacek M. Szymura

Int. J. Dev. Biol. (2013) 57: 891-895

Dual embryonic origin of the hyobranchial apparatus in the Mexican axolotl (Ambystoma mexicanum)

Asya Davidian and Yegor Malashichev

Int. J. Dev. Biol. (2013) 57: 821-828

Clonal analyses in the anterior pre-placodal region: implications for the early lineage bias of placodal progenitors

Sujata Bhattacharyya and Marianne E. Bronner

Int. J. Dev. Biol. (2013) 57: 753-757

Amphibian interorder nuclear transfer embryos reveal conserved embryonic gene transcription, but deficient DNA replication or chromosome segregation

Patrick Narbonne and John B. Gurdon

Int. J. Dev. Biol. (2012) 56: 975-986

Origins of Cdx1 regulatory elements suggest roles in vertebrate evolution

Stephen J. Gaunt and Yu-Lee Paul

Int. J. Dev. Biol. (2011) 55: 93-98

Reptile scale paradigm: Evo-Devo, pattern formation and regeneration

Cheng Chang, Ping Wu, Ruth E. Baker, Philip K. Maini, Lorenzo Alibardi and Cheng-Ming Chuong

Int. J. Dev. Biol. (2009) 53: 813-826

Proteomics analysis of regenerating amphibian limbs: changes during the onset of regeneration

Michael W. King, Anton W. Neff and Anthony L. Mescher

Int. J. Dev. Biol. (2009) 53: 955-969
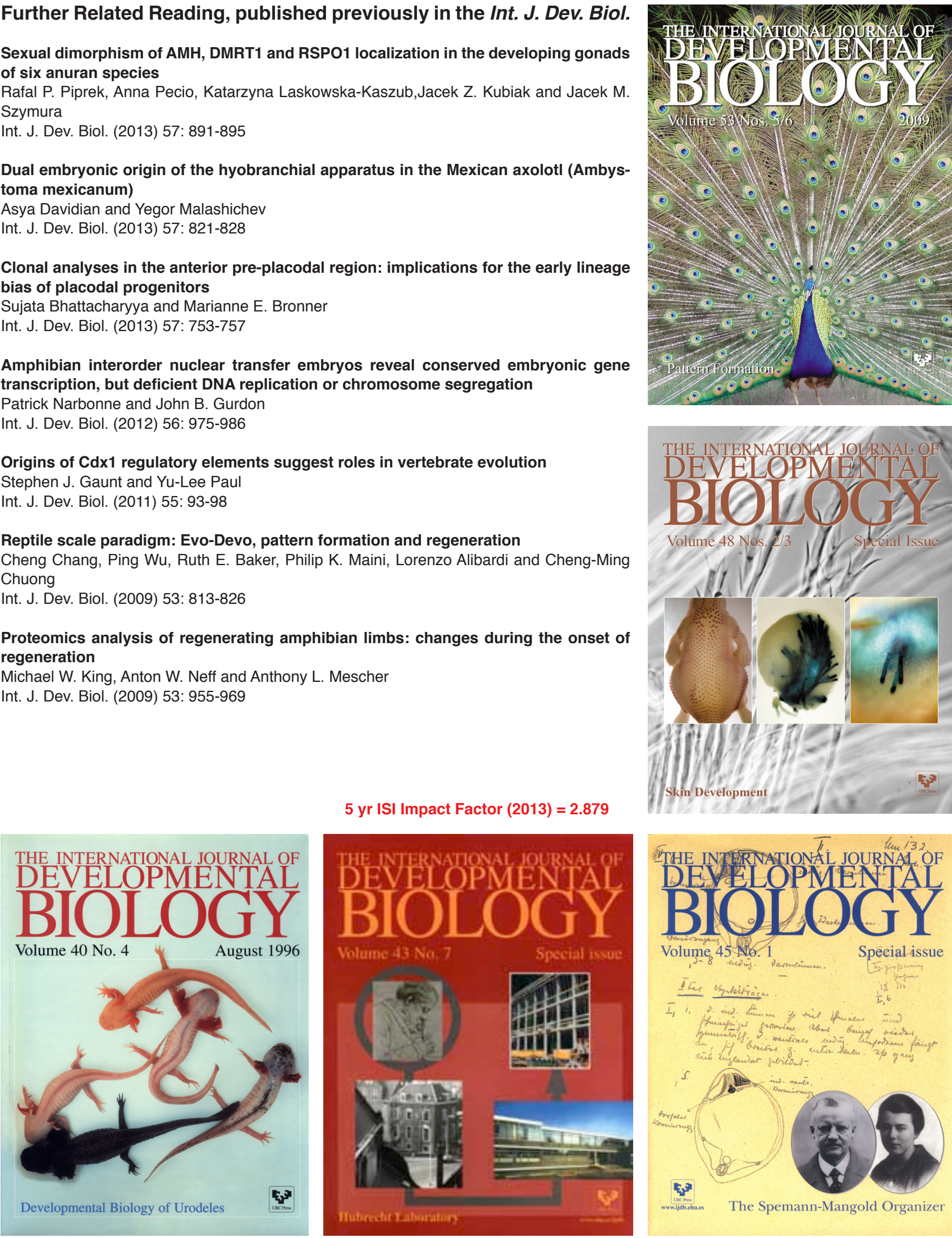

Volume 45 No. 1

Special-issue

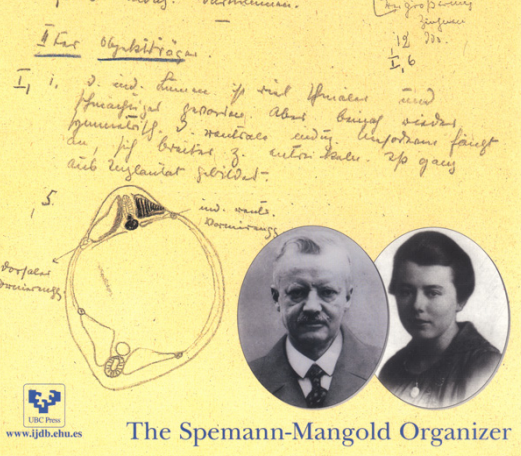

